\title{
Altered cortical activity in patients with lower limb amputation based on EEG microstate
}

\author{
Xinying Shan ${ }^{1,2, *, \dagger}$, Conghui Wei ${ }^{3, \dagger}$, Shaowen $\mathrm{Liu}^{3, \dagger}$, Jun Luo ${ }^{3, *}$ \\ ${ }^{1}$ School of Biological Science and Medical Engineering, Beihang University, 100191 Beijing, China \\ ${ }^{2}$ Beijing Key Laboratory of Rehabilitation Technical Aids for Old-Age Disability, National Research Center for Rehabilitation Technical Aids, 100176 Beijing, \\ China \\ ${ }^{3}$ Department of Rehabilitation Medicine, The Second Affiliated Hospital of Nanchang University, 330006 Nanchang, Jiangxi, China
}

*Correspondence: luojun1786@163.com (Jun Luo); shanxinying@nrcrta.cn (Xinying Shan)

${ }^{\dagger}$ These authors contributed equally.

\section{DOI:10.31083/j.jin2004100}

This is an open access article under the CC BY 4.0 license (https://creativecommons.org/licenses/by/4.0/).

Submitted: 23 August 2021 Revised: 3 October 2021 Accepted: 26 October 2021 Published: 30 December 2021

Previous studies have revealed significant changes in electroencephalogram (EEG) microstates in neuropsychiatric diseases, including schizophrenia, depression, and dementia. To explore the restingstate EEC microstate with amputation, we collected the EEC datasets from 15 patients with lower limb amputation and 20 healthy controls. Then, we analyzed the parameters of four classical EEC microstates (A-D) between the two groups. Specifically, the parameters were statistically analyzed, including duration, occurrence rate, time coverage, and transition rate. According to the results, the duration of microstate $C(t=2.95, p=0.005)$ in the lower limb amputation group was significantly smaller compared with the control group, while the occurrence rate of microstate $\mathrm{B}(t=-2.22, p=0.03)$ and $\mathrm{D}(t=-3.35, p=$ 0.002 ) were significantly larger in the lower limb amputation group. In addition, the transition rate of microstate differed significantly in AC, CA, DB between the two groups. Our results implied: (1) amputation has changed the resting-state EEG microstate; (2) EEG microstate analysis can be an approach to explore the alteration of cortical function.

\section{Keywords}

EEG microstate; Lower limb amputation; Resting-state EEC; Brain plasticity

\section{Introduction}

According to the site, amputation can be classified into upper limb amputation and lower limb amputation. Amputation usually results from vascular disease, malignant tumors, trauma, diabetes, and genetic factors [1]. Disfunction of peripheral sensory information and insufficient peripheral effector organs with amputation would inevitably induce brain plasticity. After upper limb amputation, studies have reported brain reorganization in the primary sensorimotor cortex (SMC) [2, 3].

Dettmers et al. [4] used functional magnetic resonance imaging (fMRI) to observe that the adjacent representation (the face or shoulder representation) would expand to the affected hand representation in upper limb amputees. In addition, compared with healthy controls, there was a higher activity level of the contralateral primary motor cortex (M1) in the upper limb amputees, especially of the face representation within SMC [5, 6]. Bramati et al. [7] found a weak connection between the cerebral hemispheres' functional connectivity, between the primary somatosensory areas (S1) and secondary somatosensory areas (S2), and between the primary motor areas (M1) and secondary motor areas (M2). In contrast, an enhanced connection was observed in the primary and secondary somatosensory cortex areas and the primary and premotor regions within the cerebral hemispheres. These structural and functional reorganizations in inter-hemispheric and intra-hemispheric sensory and motor cortex areas improved our understanding of brain plasticity after amputation [7]. These cortical reorganizations were interpreted as brain adaptation to the loss of peripheral sensory and motor input [8].

Brain modularity such as fMRI can analyze and evaluate cortical reorganization for its high spatial resolution $[9,10]$. Still, fMRI's relatively low time resolution makes it difficult to reflect the timing process of cortical reorganization. The electroencephalogram (EEG) with a high temporal resolution can provide a new strategy for the timing information of cortical reorganization [11]. Among all the EEG imaging technologies, we selected EEG microstate analysis with spatial and temporal characteristics due to its high temporal resolution of whole-brain cortical activities [12]. Britz et al. [13] pointed out that EEG microstate and functional magnetic resonance imaging blood oxygen level-dependent (BOLD) signals in resting-state networks are closely related but cannot fully reflect the same cortical activities.

EEG microstate corresponded for the basic unit of brain information processing, which was also considered the atom of consciousness and the smallest element of cognition [14]. Rather than randomly or continuously changing over time, the EEG microstate topographic maps seem to maintain a relatively stable state of about 60 to $120 \mathrm{~ms}$, then suddenly 
transform to new topographic maps. In the steady-state, the intensity of the topographic maps was observed to increase or decrease, similarly in the polarity. Thus, these quasi-stable periods which characterized the level of cortical activity and mental processes are called "microstates" [15].

According to the cross-validation principle, four basic EEG microstates are regarded as the optimal number of clusters. Different researches of four basic EEG microstates consistently across healthy subjects presented regular changes. Based on fMRI, the four EEG microstates revealed specific functional networks: A related to auditory, $\mathrm{B}$ related to visual, $\mathrm{C}$ related to cognitive control, and $\mathrm{D}$ connected to attention [13]. Previous studies have analyzed four parameters: duration, occurrence rate, time coverage, and transition rate to reveal brain activity in different states $[16,17]$. For example, duration was interpreted as the stability of the underlying neural components, and the occurrence rate may reflect the activated direction of the underlying neural generators [12].

EEG microstate of patients with different diseases can reflect the correlation of brain function state. Recent studies have found a higher occurrence rate of microstate $C$ and a significantly lower duration of microstates $\mathrm{B}$ and $\mathrm{D}$ in schizophrenia patients than healthy controls [18-20]. These EEG microstate findings may explain typical clinical manifestations of schizophrenia, such as insufficient attention and self-monitoring during auditory verbal hallucinations. In addition, a higher occurrence rate and larger time coverage of microstate $B$ were observed in autism spectrum disorder (ASD) compared with healthy controls [21]. These studies suggested that EEG microstate analysis can be a new approach to exploring cortical activity with potential clinical value.

Previous neuroimaging studies have characterized structural and functional reorganizations with amputation in inter-hemispheric and intra-hemispheric sensory and motor areas. Still, no research explored whether EEG microstate may be altered by amputation. Therefore, we analyzed the differences in EEG microstate parameters between patients with lower limb amputation (LLA) and healthy controls under resting state. We predict that the spatial and temporal statistical alterations would be found in specific microstate parameters in patients with LLA.

\section{Materials and methods \\ 2.1 Participants}

In order to explore the EEG microstate with amputation, we enrolled 35 right-handed subjects including 15 patients with LLA (mean age $=47.20$ years, $S D=10.00$ years; mean amputation time $=22.07$ months, $\mathrm{SD}=27.27$ months) and 20 healthy controls (mean age $=36.55$ years, $\mathrm{SD}=13.20$ years). Among the 15 lower limb amputees, 10 lower limb amputees had intermittently phantom limb pain, 4 had no phantom limb pain, and 1 had permanent phantom limb pain. None of the amputees had the experience of the prosthesis. The control group and lower limb amputees have no drug abuse and neuropsychiatric diseases history. The basic information of the amputee is shown in Table 1. The Ethics Committee has approved this experiment of the National Research Center of Rehabilitation for Technical Aids, and all participants have signed a written informed consent form before the experiment.

\subsection{EEG recording}

The experiment was conducted in a sound and electrically shielded room to reduce the noise interference during the EEG data acquisition. Before the experiment, we explained the acquisition procedures to all subjects and informed them that the experiment was harmless. Especially, the participants were required to avoid head shaking and swallowing during the EEG recording. About 5 min eyes-closed restingstate EEG data were recorded by a 256-channel EGI dense array acquisition system. The system consists of a 256-channel EEG acquisition amplifier and a 256-channel electrode cap. The $\mathrm{Cz}$ electrode was taken as a reference electrode during the recording with sampling rate set as $500 \mathrm{~Hz}$ and electrode impedance threshold below $50 \mathrm{~K} \Omega$.

\subsection{EEG data preprocessing}

The raw EEG data contained target and noise signals, such as blinks, electromyography, and electrocardiography. The quality of EEG data would significantly affect the final results so that we used Matlab2017 (Natick, MA, USA) and eeglab (EEGLAB version 14, La Jolla, CA, USA) software to preprocess the raw EEG data and eliminate noise interference [22]. Electrodes on the neck/face were excluded, and the remaining 204 electrodes were kept for further analysis [23]. Re-referencing was defined as selecting an absolute zero-potential point. Here, we assumed the average potential of the whole brain as a reference potential point, we used a $1 \mathrm{~Hz}$ high-pass filter and a $30 \mathrm{~Hz}$ low-pass filter to preserve a useful frequency band [24]. Then, we segmented the above data into $2000 \mathrm{~ms}$ epochs and removed the bad epochs by visual inspection. Finally, we retained $90 \%$ of the total epochs.

Runica, one of the Independence Component Analysis (ICA) algorithms for separating EEG signals from artifacts, was applied to remove the noise signals (i.e., blinks, electromyography, electrocardiography). After runica, we manually identified and removed the artifact components based on visual inspection. For example, blink artifact components were usually low frequency and high energy, located in the front of component maps [25].

\subsection{EEG microstate analysis}

The above pre-processing EEG data did the microstate analysis. First, we calculated the topographic maps and the Global Field Power (GFP) at all times. Then the GFP peak of topographic maps was mainly selected for later clustering to reduce the computational effort. There are two common clustering methods for EEG microstate analysis: the K-Means clustering algorithm and Topo-graphic Atomize \& Agglomerate Hierarchical Clustering (T-AAHC) algorithm. K-Means clustering algorithm was conducted via the following steps: (1) Randomly selecting $\mathrm{K}$ topographic maps as the 
Table 1. The basic information of patients with LLA.

\begin{tabular}{|c|c|c|c|c|c|c|}
\hline Patient & Gender & Age (year) & Amputation location & Amputation time (month) & Reason & Phantom limb pain \\
\hline 1 & Female & 57 & Shank & 8 & Accident & Intermittent \\
\hline 2 & Male & 46 & Thigh & 4 & Accident & Intermittent \\
\hline 3 & Male & 41 & Shank & 7 & Accident & Intermittent \\
\hline 4 & Male & 49 & Shank & 24 & Accident & Intermittent \\
\hline 5 & Male & 49 & Thigh & 2 & Accident & Absence \\
\hline 6 & Male & 53 & Thigh & 5 & Burn & Intermittent \\
\hline 7 & Male & 32 & Thigh & 72 & Electrical burn & Absence \\
\hline 8 & Male & 52 & Shank & 1 & Genetic disease & Intermittent \\
\hline 9 & Female & 66 & Thigh & 11 & Infection & Intermittent \\
\hline 10 & Male & 35 & Shank & 5 & Infection & Absence \\
\hline 11 & Male & 46 & Shank & 22 & Accident & Intermittent \\
\hline 12 & Male & 32 & Shank & 4 & Accident & Absence \\
\hline 13 & Male & 45 & Shank & 47 & Accident & Intermittent \\
\hline 14 & Male & 62 & Shank & 90 & Accident & Intermittent \\
\hline 15 & Male & 44 & Thigh & 29 & Tumor & Permanent \\
\hline
\end{tabular}

initial cluster centers; (2) Calculating the distance of each topographic map and cluster centers, then assigning the topographic maps to the closest cluster center; (3) Obtaining a new cluster center by updating iteratively according to topographic maps and repeating (1) and (2) until reaching end condition.

Since seed clusters of this k-means clustering algorithm were based on different clustering results in every run, and the initial prototype maps were randomly selected, the microstates could be slightly different in each operation. Thus, this $\mathrm{k}$-means clustering algorithm generally was repeated several dozen times, which profoundly increased computational effort. For this reason, the T-AAHC algorithm was selected. Compared with the K-Means clustering algorithm, T-AAHC regarded each topographic map as a cluster that operated in a down-top manner. Then by merging the two closest clusters into a new one, the number of topographic maps decreased from the predefined maximum to 1 . Other literature has provided detailed information about this algorithm [26-28].

The initial topographic maps at multiple moments could be clustered into several representative ones through the clustering algorithm. Grand mean model maps were very similar to the basic four maps, and the latter were labeled into 'microstate classes', such as 'A', 'B', 'C' and 'D' [29]. In addition, the primary four maps fit all initial prototype maps with high Global Explained Variance (GVE), which explained the appropriate level. Thus, we used the basic four maps to compute microstates parameters of each subject. The parameters were often statistically analyzed, including duration, occurrence rate, time coverage, and transition rate, which reflected the information of neural activities: (1) the microstate duration indicated the stability of underlying neural components. It was defined as a mean length of the sequence milliseconds. First, all the identical and adjacent initial prototype maps were assigned to one sequence. Then the sequence was calculated from the first initial prototype map to the last one;
(2) the occurrence rate indicated an activated trend of the underlying neural generator and was defined as the number of occurrences of each microstate per second; (3) the time coverage defined as the time of a microstate as a relative percentage of the total time; (4) the transition rate reflected the probability of a given microstate transforming to others randomly, and explained the activation sequence of a neural generator.

\subsection{Statistical analysis}

To verify our hypothesis that the EEG microstates may be significantly different between the LLA group and healthy controls, a $t$-test measure was performed simultaneously by comparing four microstates. We applied false discovery rate (FDR) correction for each $p$-value to reduce the type I error rate. The statistical significance defaulted as $p<0.05$ for the test, and the alpha value for FDR results was set as 0.05. All statistical analyses were performed in SPSS v17 for Macintosh (IBM Corporation, Armonk, NY, USA).

\section{Results}

According to calculation results, the final microstate topographic maps of the LLA and HC groups are shown in Fig. 1 (Ref. [30]). MS1, MS2, MS3, and MS4 correspond to microstate A, B, C, and D. The topographic map gravity of four microstates is as: microstate $A$ indicated rightanterior to left-posterior orientation, microstate B indicated left-anterior to right-posterior orientation, microstate $\mathrm{C}$ indicated anterior-posterior orientation and microstate $\mathrm{D}$ indicated front-central extreme, respectively. The alteration in the spatial distribution of primary neuronal sources indicates a significant difference in the map configuration between the LLA group and healthy controls for microstate C and D.

The $t$-test results of resting-state EEG microstate parameters of the two groups are shown in Table 2. For the duration of microstates, $t$-test implied that the healthy controls (duration $=59.66 \mathrm{~ms})$ spent more time in microstate $\mathrm{C}(t=2.95$, 
LLA
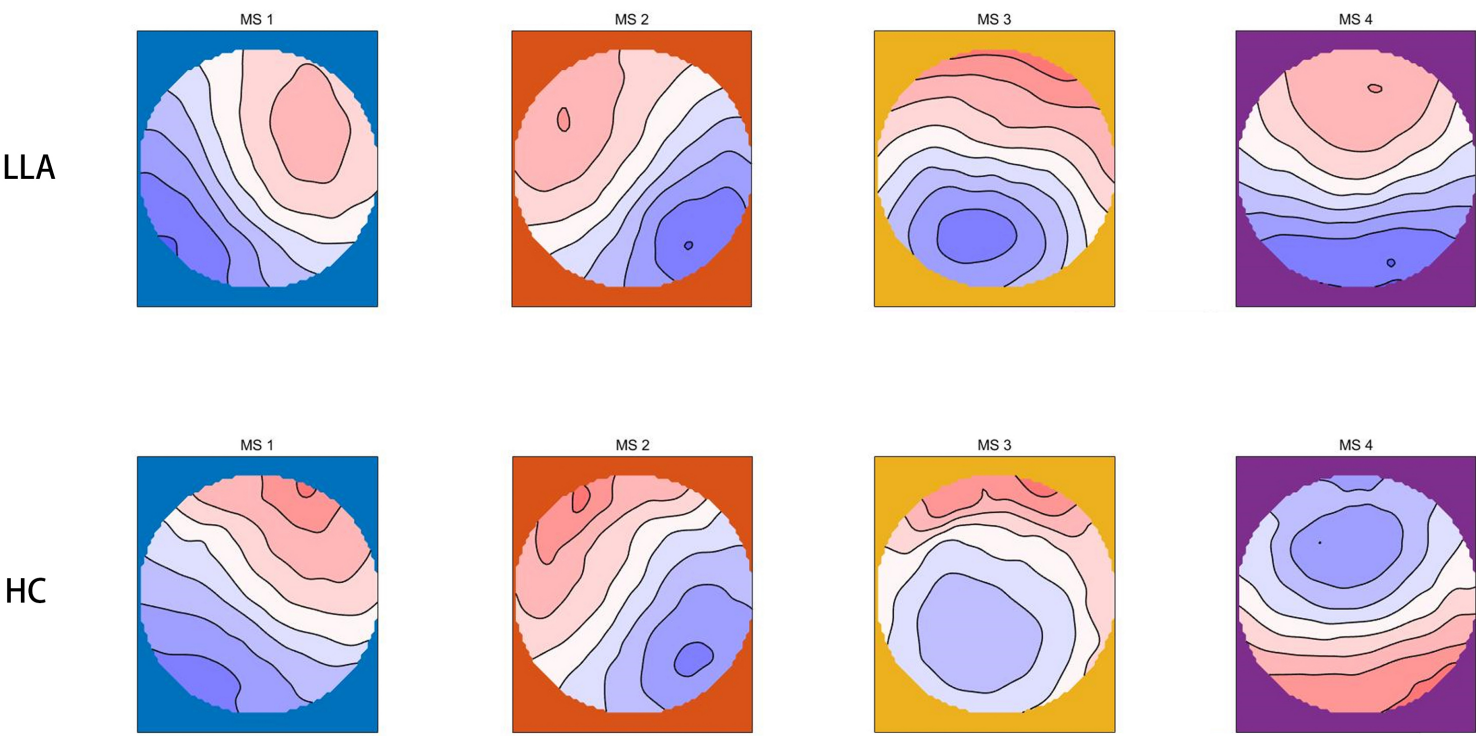

Fig. 1. Microstate topographic maps of patients with LLA and HC controls. MS1, MS2, MS3 and MS4 correspond to microstate classes A, B, C, and D, respectively. Koenig et al. [30] found that four microstates could explain around 79\% of cortical activity.

$p=0.005)$ compared with the lower limb amputees (duration $=51.59 \mathrm{~ms})$. No differences were observed for microstate $\mathrm{A}$ $(t=0.99, p=0.33)$, microstate $\mathrm{B}(t=0.29, p=0.76)$ and microstate $\mathrm{D}(t=1.63, p=0.11)$. For microstate occurrence rate, $t$-test found that microstate $\mathrm{B}(t=-2.22, p=0.03)$ and $\mathrm{D}$ $(t=-3.35, p=0.002)$ were significantly more frequent in the LLA group compared with healthy controls. No differences were observed for microstate A $(t=-1.14, p=0.25)$ and $\mathrm{C}(t$ $=0.71, p=0.48)$. Lastly, all $t$-test results of the time coverage for four microstates were $p>0.05$ which indicated no significant differences between healthy control group and the LLA group.

For microstate transition rate (see Table 3), the two groups displayed a transformed tendency of concatenations between microstate $\mathrm{A}$ and $\mathrm{C}$. The result clarified that the transitions rate from microstate $\mathrm{D}$ to microstate $\mathrm{B}(p=0.04)$ differed significantly with directional predominance between the two groups. Still, there was no significant difference from microstate B to microstate D $(p=0.051)$. Moreover, no significant differences were noted at other transition rate types.

\section{Discussion}

In this short communication, we used an EEG microstate to analyze the resting-state EEG of lower limb amputees. Then, we observed a lower duration in microstate $\mathrm{C}$ and $\mathrm{a}$ higher frequency in microstate B and $\mathrm{D}$ in lower limb amputees compared with healthy controls. In addition, the transition rate from microstate $\mathrm{A}$ to microstate $\mathrm{C}$ differed significantly in directional predominance between the two groups. These results indicated that the EEG microstate parameters of visual processing related to microstate $B$ and attention processing related to microstate $\mathrm{D}$ might be altered in patients with LLA.
Table 2. Statistical results of microstate parameters between patients with LLA and HC controls.

\begin{tabular}{lcccc}
\hline Parameter & Patients & Controls & $t$-value & $p$-value \\
\hline Duration (ms) & & & & \\
A & 56.88 & 59.31 & 0.99 & 0.33 \\
B & 59.49 & 60.26 & 0.29 & 0.76 \\
C & 51.59 & 59.66 & 2.95 & $0.005^{*}$ \\
D & 57.04 & 61.61 & 1.63 & 0.11 \\
Occurrence rate & & & & \\
A & 4.36 & 4.17 & -1.14 & 0.25 \\
B & 4.89 & 4.43 & -2.22 & $0.03^{*}$ \\
C & 3.71 & 4.00 & 0.71 & 0.48 \\
D & 4.70 & 3.96 & -3.35 & $0.002^{*}$ \\
Time coverage & & & & \\
A & 0.25 & 0.25 & -0.16 & 0.87 \\
B & 0.28 & 0.27 & -1.33 & 0.19 \\
C & 0.20 & 0.24 & 1.74 & 0.09 \\
D & 0.27 & 0.24 & -1.10 & 0.27 \\
\hline
\end{tabular}

The table showed the duration in milliseconds (ms), occurrence rate, time coverage of four microstate classes (A-D). Significant level was ${ }^{*} p<0.05$.

Table 3. Statistical results of microstate transition rate between patients with LLA and HC controls.

\begin{tabular}{lccc}
\hline Transition types & $p$-value & Transition types & $p$-value \\
\hline From A to B & 0.38 & From C to A & $0.005^{*}$ \\
From A to C & $0.007^{*}$ & From C to B & 0.09 \\
From A to D & 0.11 & From C to D & 0.79 \\
From B to A & 0.36 & From D to A & 0.08 \\
From B to C & 0.11 & From D to B & $0.04^{*}$ \\
From B to D & 0.051 & From D to C & 0.66 \\
\hline Significant level was & * $<0.05$ &
\end{tabular}

Significant level was ${ }^{*} p<0.05$. 
Wife et al. [31] found that losing a limb or afferent nerve conduction limb could induce functional or structural reorganization in visual areas. Compared with healthy controls, lower limb amputees have observed significantly lower thickness in the V5/middle temporal (V5/MT+) visual area and the same cortical alteration in the V3d. However, no significant alterations in other visual areas were observed between the two groups [32]. In addition, Palermo et al. [33] found that the loss of peripheral sensor and motor inputs in patients with right lower amputation would reduce the ability to recognize the relationship between different body parts, which was the same as the loss of up-down information in patients with brain damage due to personal negligence. Visual and spatial mental representations of one's body arise from peripheral information about our body rather than visually processing other people's body information. Body representations are not entirely hard-wired in the brain $[33,34]$. These results are consistent with ours, indicating that lower-limb amputees may be altered in the visual processing network.

Microstate $\mathrm{D}$ was associated with negative BOLD signals in the frontal and parietal cortex's right-lateralized dorsal and ventral areas [13]. Ventral frontoparietal regions subserve reflexive aspects of attention such as detecting behavioral stimuli, while more dorsal regions in the frontoparietal cortex are related to conversion and redirection of attention [35]. Patients with upper limb amputation showed difficulties in mental rotation of body parts, though they could still imagine the lost limb movements [36]. Compared with healthy controls, the amputation group displayed a lack of difference between imagery modalities (visual and kinesthetic). However, the Kinesthetic and Visual Imagery Questionnaire (KVIQ) scores tended to be lower, suggesting a relatively lower performance in visual imagery after amputation [37].

Nico et al. [38] found that patients with upper limb amputation performed much more slowly and less accurately than healthy controls when participating in an imagery handedness judgment task. Researchers concluded that losing a limb would not stop motor imagery but would significantly enhance its difficulty. Scandola and his colleagues [39] found that the individuals with spinal cord injury performed the sensorimotor "bio-mechanical effect" (orientation-dependent modulation of response times) for the mental rotation of foot images after physiotherapy. It seems reasonable that sensory deprivation is associated with aberrancies in sensory-motor processing due to altered microstates. Based on these studies, we can speculate that the loss of peripheral information relevant to the limb can activate the attention network effectively and induce brain reorganization.

Microstate $\mathrm{C}$ was associated with cognitive control and correlated with positive BOLD activation in the posterior part of the anterior cingulate cortex and bilateral inferior frontal gyri, the right anterior insula and the left claustrum $[13,23]$. Especially, a close relation was observed in an independent component between microstate $\mathrm{C}$ and positive
BOLD in the insula. The insular cortex was considered part of the saliency network and played a crucial role in alternating the central-executive function and the default mode network (DMN) [40]. Besides, microstate $\mathrm{C}$ is considered to be related to the subjective representation of one's own body by combining interoceptive information with affective salience, were significantly positively correlated with the disgust sensitivity level [23]. Direct source localization of EEG microstates confirmed the implication of frontal brain regions in the generation of microstate $\mathrm{C}$. These regions were attributed to the anterior parts of the default mode network [41]. The physiological basis of microstate $\mathrm{C}$ is still highly debated. Therefore, further work is needed for microstate $\mathrm{C}$.

For microstate A, Mantini et al. [42] found that the relationship between microstate $\mathrm{A}$ and negative BOLD activation predominately in bilateral superior and middle temporal gyri areas was interpreted as phonological processing. In addition, a close temporal and spatial correlation with an independent component between microstate $A$ and negative BOLD activation was observed [13, 43]. Since amputees rarely displayed abnormalities in the phonological processing network, there was no significant difference of microstate A between the LLA group and healthy controls.

Neurophysiology researches have proved that limb amputation is a strong driver of brain plasticity $[3,4,10]$. Brain plasticity has two implications: (1) The cortex near the damaged cortex can replace the latter's functions to the full extent; (2) Sensorimotor cortex will respond to afferents from residual limbs after amputation. Brain plasticity may have two concerning mechanisms: forming new synaptic contact or strengthening the existing synaptic contact. The structure of brain plasticity features the shape, orientation, size, and several inhibitory or excitatory synapses in specific areas [44]. Brain plasticity has been accepted as a sound theoretical basis for some therapies such as rehabilitation after stroke, transcranial magnetic stimulation, and deep brain stimulation. For example, transcranial direct current stimulation of Broca's area increase cognitive-verbal performance by modulating brain electrical activity in language-related regions [45]. Thus, harnessing the potential of this endogenous biological self-repairing process and restoring brain functionality in the context of acute and chronic neurological diseases has been one of the key promises for translational therapeutic strategies [46].

Mizuguchi et al. [47] reported that motor imagery vividness in the amputated limb was enhanced by walking with a prosthesis, which implied that using a prosthesis would preserve the mental representation of a missing limb. When the long jumper from a long jump Paralympic gold medalist with a unilateral below-knee amputation was asked to perform a rhythmic isometric contraction of knee extensor muscles with the take-off/prosthetic leg, they observed contralateral M1/S1, and ipsilateral M1/S1 were activated [47]. In our experiment, none of the amputees had the experience of the prosthesis. Therefore, our results cannot explain whether 
prosthesis will affect the EEG microstate or not after amputation. For phantom limb pain, 10 lower limb amputees had phantom limb pain intermittently, 4 had no phantom limb pain, and 1 had phantom limb pain permanently. Several studies described brain plasticity in people with amputation who did not have phantom limb pain [10,48]. However, brain plasticity occurred in the presence of phantom pain, mainly at the primary and somatosensory motor cortex levels $[49,50]$.

Moreover, previous studies found a negative correlation between phantom limb pain and decreased bilateral visual cortex thickness. Visual feedback training on the residual limb can reduce phantom limb pain intensity, which may be due to the activation of mirror neurons in the hemisphere of the brain that is contralateral to the residual limb $[34,51]$. The high-density 256-channels EGI device could contain richer spatial source information. In future studies, we would apply a LORATE [52] or dipole analysis [53] to estimate the source of each microstate signal.

\section{Conclusions}

We analyzed the parameters of the resting-state EEG microstate between patients with lower limb amputation and healthy controls to investigate whether amputation could alter intrinsic cortical activities or not. We found that patients with lower limb amputation had significantly lower duration for microstate $\mathrm{C}$ and a larger occurrence rate of microstate $\mathrm{B}$ and $\mathrm{D}$ than healthy controls. Furthermore, it indicated that loss of peripheral sensor and motor inputs could induce brain plasticity. The resting-state EEG microstate analysis can be an approach to explore the mechanism of cortical reorganization.

\section{Abbreviations}

ADHD, attention deficit hyperactivity disorder; LLA, lower limb amputation; HC, healthy controls; SMC, primary sensorimotor cortex; fMRI, Functional magnetic resonance imaging; EEG, electroencephalogram; M1, primary motor cortex; S1, primary somatosensory areas; S2, secondary somatosensory areas; M2, secondary motor areas; GFP, Global Field Power; GVE, Global Explained Variance; $\mathrm{V} 5 / \mathrm{MT}+, \mathrm{V} 5 /$ middle temporal; BOLD, blood oxygen leveldependent; ICA, independent component analysis; ASD, autism spectrum disorder; T-AAHC, Topo-graphic Atomize \& Agglomerate Hierarchical Clustering; FDR, false discovery rate; $\mathrm{DMN}$, default mode network.

\section{Author contributions}

XS conceived and designed the experiments; CW performed the experiments; SL analyzed the data; JL contributed reagents and materials; CW and XS wrote the paper.

\section{Ethics approval and consent to participate}

The Ethics Committee approved the present research of laboratory animals of the Nation Research Center of Reha- bilitation for Technical Aids (NRRA2020038). We obtained the informed consent form of all participants.

\section{Acknowledgment}

Not applicable.

\section{Funding}

This research was supported by National Key R\&D Program of China (2020YFC2005801).

\section{Conflict of interest}

The authors declare no conflict of interest.

\section{References}

[1] Varma P, Stineman MG, Dillingham TR. Epidemiology of Limb Loss. Physical Medicine and Rehabilitation Clinics of North America. 2014; 25: 1-8

[2] Karl A, Mühlnickel W, Kurth R, Flor H. Neuroelectric source imaging of steady-state movement-related cortical potentials in human upper extremity amputees with and without phantom limb pain. Pain. 2004; 110: 90-102.

[3] Makin TR, Cramer AO, Scholz J, Hahamy A, Henderson Slater D Tracey I, et al. Deprivation-related and use-dependent plasticity go hand in hand. eLife. 2013; 2: e01273.

[4] Dettmers C, Adler T, Rzanny R, van Schayck R, Gaser C, Weiss T, et al. Increased excitability in the primary motor cortex and supplementary motor area in patients with phantom limb pain after upper limb amputation. Neuroscience Letters. 2001; 307: 109-112.

[5] G Hordacre B, C Ridding M, V Bradnam L. The potential for noninvasive brain stimulation to improve function after amputation. Disability and Rehabilitation. 2016; 38: 1521-1532.

[6] Elbert T, Rockstroh B. Reorganization of human cerebral cortex the range of changes following use and injury. The Neuroscientist. 2004; 10: 129-141.

[7] Bramati IE, Rodrigues EC, Simões EL, Melo B, Höfle S, Moll J, et al Lower limb amputees undergo long-distance plasticity in sensorimotor functional connectivity. Scientific Reports. 2019; 9: 2518.

[8] Flor H, Diers M, Andoh J. The neural basis of phantom limb pain. Trends in Cognitive Sciences. 2013; 17: 307-308.

[9] Pawela CP, Biswal BB, Hudetz AG, Li R, Jones SR, Cho YR, et al. Interhemispheric neuroplasticity following limb deafferentation detected by resting-state functional connectivity magnetic resonance imaging (fcMRI) and functional magnetic resonance imaging (fMRI). NeuroImage. 2010; 49: 2467-2478.

[10] Simões EL, Bramati I, Rodrigues E, Franzoi A, Moll J, Lent R, et al. Functional expansion of sensorimotor representation and structural reorganization of callosal connections in lower limb amputees. The Journal of Neuroscience. 2012; 32: 3211-3220.

[11] Yuanyuan Lyu, Xiaoli Guo, Zhuo Wang, Shanbao Tong. Restingstate EEG network change in alpha and beta bands after upper limb amputation. Annual International Conference of the IEEE Engineering in Medicine and Biology Society. 2016; 2016: 49-52.

[12] Michel CM, Koenig T. EEG microstates as a tool for studying the temporal dynamics of whole-brain neuronal networks: A review. NeuroImage. 2018; 180: 577-593.

[13] Britz J, Van De Ville D, Michel CM. BOLD correlates of EEG topography reveal rapid resting-state network dynamics. NeuroImage. 2010; 52: 1162-1170.

[14] Lehmann D, Ozaki H, Pal I. EEG alpha map series: brain micro-states by space-oriented adaptive segmentation. Electroencephalography and Clinical Neurophysiology. 1987; 67: 271-288.

[15] Khanna A, Pascual-Leone A, Michel CM, Farzan F. Microstates in resting-state EEG: current status and future directions. Neuroscience and Biobehavioral Reviews. 2015; 49: 105-113. 
[16] Bréchet L, Brunet D, Birot G, Gruetter R, Michel CM, Jorge J. Capturing the spatiotemporal dynamics of self-generated, taskinitiated thoughts with EEG and fMRI. NeuroImage. 2019; 194: 82-92.

[17] Drissi NM, Szakács A, Witt ST, Wretman A, Ulander M, Ståhlbrandt $\mathrm{H}$, et al. Corrigendum: Altered Brain Microstate Dynamics in Adolescents With Narcolepsy. Frontiers in Human Neuroscience. 2019; 13: 385.

[18] Andreou C, Faber PL, Leicht G, Schoettle D, Polomac N, Hanganu-Opatz IL, et al. Resting-state connectivity in the prodromal phase of schizophrenia: insights from EEG microstates. Schizophrenia Research. 2014; 152: 513-520.

[19] Lehmann D, Faber PL, Galderisi S, Herrmann WM, Kinoshita $\mathrm{T}$, Koukkou M, et al. EEG microstate duration and syntax in acute, medication-naive, first-episode schizophrenia: a multicenter study. Psychiatry Research. 2005; 138: 141-156.

[20] Rieger K, Diaz Hernandez L, Baenninger A, Koenig T. 15 Years of Microstate Research in Schizophrenia - Where Are We? A MetaAnalysis. Frontiers in Psychiatry. 2016; 7: 22.

[21] Jia H, Yu D. Aberrant Intrinsic Brain Activity in Patients with Autism Spectrum Disorder: Insights from EEG Microstates. Brain Topography. 2019; 32: 295-303.

[22] Delorme A, Makeig S. EEGLAB: an open source toolbox for analysis of single-trial EEG dynamics including independent component analysis. Journal of Neuroscience Methods. 2004; 134: 9-21.

[23] Tomescu MI, Rihs TA, Rochas V, Hardmeier M, Britz J, Allali G, et al. From swing to cane: Sex differences of EEG resting-state temporal patterns during maturation and aging. Developmental Cognitive Neuroscience. 2018; 31: 58-66.

[24] Britz J, Díaz Hernàndez L, Ro T, Michel CM. EEG-microstate dependent emergence of perceptual awareness. Frontiers in Behavioral Neuroscience. 2014; 8: 163.

[25] Islam MK, Rastegarnia A, Yang Z. Methods for artifact detection and removal from scalp EEG: A review. Neurophysiologie Clinique. 2016; 46: 287-305.

[26] Lehmann D, Skrandies W. Reference-free identification of components of checkerboard-evoked multichannel potential fields. Electroencephalography and Clinical Neurophysiology. 1980; 48: 609-621.

[27] Van de Ville D, Britz J, Michel CM. EEG microstate sequences in healthy humans at rest reveal scale-free dynamics. Proceedings of the National Academy of Sciences of the United States of America. 2010; 107: 18179-18184.

[28] Skrandies W. Global field power and topographic similarity. Brain Topography. 1990; 3: 137-141.

[29] Seitzman BA, Abell M, Bartley SC, Erickson MA, Bolbecker AR, Hetrick WP. Cognitive manipulation of brain electric microstates. NeuroImage. 2017; 146: 533-543.

[30] Koenig T, Prichep L, Lehmann D, Sosa PV, Braeker E, Kleinlogel $\mathrm{H}$, et al. Millisecond by millisecond, year by year: normative EEG microstates and developmental stages. NeuroImage. 2002; 16: 4148.

[31] Wilf M, Holmes NP, Schwartz I, Makin TR. Dissociating between object affordances and spatial compatibility effects using early response components. Frontiers in Psychology. 2013; 4: 591.

[32] Jiang G, Li C, Wu J, Jiang T, Zhang Y, Zhao L, et al. Progressive Thinning of Visual Motion Area in Lower Limb Amputees. Frontiers in Human Neuroscience. 2016; 10: 79.

[33] Palermo L, Di Vita A, Piccardi L, Traballesi M, Guariglia C. Bottom-up and top-down processes in body representation: a study of brain-damaged and amputee patients. Neuropsychology. 2014; 28: 772-781.

[34] Kim SY, Kim YY. Mirror therapy for phantom limb pain. The Korean Journal of Pain. 2012; 25: 272-274.

[35] Corbetta M, Shulman GL. Control of goal-directed and stimulusdriven attention in the brain. Nature Reviews Neuroscience. 2002; 3: 201-215.
[36] Boccia M, Di Vita A, Palermo L, Nemmi F, Traballesi M, Brunelli $S$, et al. Neural modifications in lower limb amputation: an fMRI study on action and non-action oriented body representations. Brain Imaging and Behavior. 2020; 14: 416-425.

[37] Malouin F, Richards CL, Durand A, Descent M, Poiré D, Frémont $P$, et al. Effects of Practice, Visual Loss, Limb Amputation, and Disuse on Motor Imagery Vividness. Neurorehabilitation and Neural Repair. 2009; 23: 449-463.

[38] Nico D, Daprati E, Rigal F, Parsons L, Sirigu A. Left and right hand recognition in upper limb amputees. Brain. 2004; 127: 120-132.

[39] Scandola M, Dodoni L, Lazzeri G, Arcangeli CA, Avesani R, Moro $\mathrm{V}$, et al. Neurocognitive Benefits of Physiotherapy for Spinal Cord Injury. Journal of Neurotrauma. 2019; 36: 2028-2035.

[40] Milz P, Faber PL, Lehmann D, Koenig T, Kochi K, PascualMarqui RD. The functional significance of EEG microstatesAssociations with modalities of thinking. NeuroImage. 2016; 125 : 643-656.

[41] Li Z, Li Y, Li X, Zou F, Wang Y, Wu X, et al. The spontaneous brain activity of disgust: Perspective from resting state fMRI and resting state EEG. Behavioural Brain Research. 2021; 403: 113135.

[42] Mantini D, Perrucci MG, Del Gratta C, Romani GL, Corbetta M. Electrophysiological signatures of resting state networks in the human brain. Proceedings of the National Academy of Sciences of the United States of America. 2007; 104: 13170-13175.

[43] Gotman J. Epileptic networks studied with EEG-fMRI. Epilepsia. 2008; 49: 42-51.

[44] Batool S, Raza H, Zaidi J, Riaz S, Hasan S, Syed NI. Synapse formation: from cellular and molecular mechanisms to neurodevelopmental and neurodegenerative disorders. Journal of Neurophysiology. 2019; 121: 1381-1397.

[45] Saadi ZK, Saadat M, Kamali AM, Yahyavi SS, Nami M. Electrophysiological modulation and cognitive-verbal enhancement by multi-session Broca's stimulation: a quantitative EEG transcranial direct current stimulation based investigation. Journal of Integrative Neuroscience. 2019; 18: 107-115.

[46] Gatto RG, Asakawa T. New molecular insights, innovative technologies, and medical approaches in the "Exploration of mechanisms in cortical plasticity". Journal of Integrative Neuroscience. 2020; 19: 733-734.

[47] Mizuguchi N, Nakagawa K, Tazawa Y, Kanosue K, Nakazawa K. Functional plasticity of the ipsilateral primary sensorimotor cortex in an elite long jumper with below-knee amputation. NeuroImage: Clinical. 2019; 23: 101847.

[48] Jiang G, Yin X, Li C, Li L, Zhao L, Evans AC, et al. The Plasticity of Brain Gray Matter and White Matter following Lower Limb Amputation. Neural Plasticity. 2015; 2015: 823185.

[49] Irlbacher K, Meyer B, Voss M, Brandt SA, Röricht S. Spatial reorganization of cortical motor output maps of stump muscles in human upper-limb amputees. Neuroscience Letters. 2002; 321: 129132.

[50] Zhang J, Zhang Y, Wang L, Sang L, Li L, Li P, et al. Brain Functional Connectivity Plasticity within and beyond the Sensorimotor Network in Lower-Limb Amputees. Frontiers in Human Neuroscience. 2018; 12: 403.

[51] Desmond DM, Maclachlan M. Prevalence and characteristics of phantom limb pain and residual limb pain in the long term after upper limb amputation. International Journal of Rehabilitation Research. 2010; 33: 279-282.

[52] Pascual-Marqui RD, Michel CM, Lehmann D. Low resolution electromagnetic tomography: a new method for localizing electrical activity in the brain. International Journal of Psychophysiology. 1994; 18: 49-65.

[53] Scherg M, Von Cramon D. Evoked dipole source potentials of the human auditory cortex. Electroencephalography and Clinical Neurophysiology. 1986; 65: 344-360. 